Pathologe 2019 · 40:582

https://doi.org/10.1007/s00292-019-00686-7

๑) Springer Medizin Verlag GmbH, ein Teil von Springer Nature 2019

Seltene Erkrankungen waren im vergangenen Jahr ein Thema der 102. Jahrestagung der Deutschen Gesellschaft für Pathologie. Sie werden manchmal als Waisen der Medizin betrachtet, liefern aber häufig besondere Einblicke in zell- und gewebebiologische Prozesse. Der in 2013 veröffentlichte Nationale Aktionsplan des Nationalen Aktionsbündnisses für Menschen mit Seltenen Erkrankungen (NAMSE) verfolgte als übergeordnetes Ziel, die Behandelnden und die Behandelten besser zu informieren. Die medizinischen Versorgungsstrukturen sollten dabei ausgebaut und Kompetenzen gebündelt werden. Der Themenschwerpunkt „Seltene Erkrankungen“ griff diese übergeordnete Initiative auf und schuf auf der Jahrestagung in 2018 dafür ein Forum. Spannende Beiträge fanden dazu bereits ihren Niederschlag im Verhandlungsband und bekommen noch einmal ein Forum in diesem Themenheft von Der Pathologe.

Der Beitrag von R. Büttner und $N$. Friedrichs widmet sich dem erblichen Darmkrebs bei Lynch-/HNPCCSyndrom in Deutschland. Auch wenn das Lynch-Syndrom für weniger als $7 \%$ aller kolorektalen Krebserkrankungen verantwortlich ist, so muss bei allen primär diagnostizierten kolorektalen Adenokarzinomen überprüft werden, ob die revidierten Bethesda-Kriterien erfüllt sind und eine immunhistochemische und molekularpathologische Testung notwendig sind. Der Beitrag liefert eine sehr lesenswerte Zusammenfassung des Syndroms mit Kennzahlen der 18-jährigen Arbeit des Deutschen HNPCC-Konsortiums. Seltene Erkrankungen brauchen Ausdauer, Netzwerkstrukturen und liefern am Ende wichtige Informationen, die

\title{
Christoph Röcken
}

Institut für Pathologie, Christian-Albrechts-Universität, Kiel, Deutschland

\section{Seltene Erkrankungen}

in S3-Leitlinienempfehlungen münden und die Routinediagnostik beeinflussen.

Dutzmann et al. liefern ein Update zum Li-Fraumeni-Syndrom. Diese seltene Erkrankung wurde sehr bewusst ausgewählt, weil die immer häufiger zum Einsatz kommenden Tiefensequenzierungen TP53-Mutationen nachweisen und der Verdacht auf das Vorliegen eines Li-Fraumeni-Syndroms geäußert werden kann. Der Beitrag illustriert sehr anschaulich das breite Spektrum des Krankheitsbildes und auch, bei welchen Tumorentitäten besonders an Li-Fraumeni-Syndrom gedacht werden sollte. Auf 3 kleine Passagen möchte ich hier besonders aufmerksam machen: das TP53Keimbahnmosaik mit dem Risiko eines falsch negativen Befundes, der Fallstrick klonale Hämatopoese mit dem Risiko einer falsch positiven Interpretation als TP53-Keimbahnmutation und die Besonderheiten bei der Behandlung von Patienten mit Li-Fraumeni-Syndrom. Strahlentherapie birgt bei Patienten mit Li-Fraumeni-Syndrom das Risiko, Zweitund Drittneoplasien zu induzieren.

C. Vokuhl geht auf seltene kindliche Nierentumoren ein. Das sind in diesem Beitrag die Klarzellsarkome, die mesoblastischen Nephrome, die malignen Rhabdoidtumoren und das anaplastisches Sarkom. Höhn et al. beschreiben einen Fall des seltenen un- und dedifferenzierten Endometriumkarzinoms. Es handelt sich um klinisch aggressive Tumoren des mittleren Alters. Beide Beiträge beschäftigen sich mit seltenen Tumorentitäten und liefern wichtige Hinweise auf immunhistochemische und molekularpathologische Stufendiagnostik.

Schließlich rundet ein Beitrag von S. Detlefsen zu den IgG4-assoziierten Erkrankungen dieses Themenheft ab.
IgG4-assoziierte Erkrankungen treten an verschiedenen Stellen des Organismus auf und sind vor allem in der Biopsiediagnostik manchmal schwierig zu fassen. Der Beitrag liefert neben nützlichen diagnostischen Algorithmen auch Einblicke in die Pathophysiologie.

Die hier zusammengefasste Auswahl kann nicht das große Spektrum seltener Erkrankungen abdecken. Das Themenheft soll aber den Nachwuchs ermuntern, Themen außerhalb des Forschungsmainstreams aufzugreifen. Dies kommt in hohem Maße den Patienten ${ }^{\star}$ innen mit seltenen Erkrankungen zu Gute, die von einer verbesserten Diagnostik und Therapie profitieren.

\section{Korrespondenzadresse}

Prof. Dr. Christoph Röcken

Institut für Pathologie, Christian-AlbrechtsUniversität

Arnold-Heller-Str. 3/Haus U33, 24105 Kiel, Deutschland

christoph.roecken@uksh.de

Interessenkonflikt. C. Röcken gibt an, dass kein Interessenkonflikt besteht. 